\title{
Enhancing Adaptability of Foreign Students
}

Olga G. Arkatova

\author{
Nikolay S. Danakin
}

Irina V. Shavyrina*

Belgorod State Technological University named after V.G. Shukhov, Russian Federation

*Corresponding Email: shavyrina_77@mail.ru

\section{Doi:10.5901/mjss.2015.v6n6s7p276}

\begin{abstract}
The article is devoted to the study and analysis of foreign students' adaptability in Russian universities. Based on the analysis of the structured adaptation readiness of an individual, as well as features of the socio-cultural adaptation of foreign students, the study determines the structure of adaptation readiness, which includes such components as cognitive, informational, evaluative, motivational, dispositional, emotional, volitional, operational, communicative, and socio-psychological. Analysis of empirical data reveal the main problems and prospects to improve the adaptation readiness of foreign students.
\end{abstract}

Keywords: international student, adaptation readiness, socio-cultural adaptation

\section{Introduction}

Nowadays, the national higher education system of the Russian Federation is undergoing a process of integration into the world's high school and academic community. The effective deployment of intelligent communications and competencebased approach are being implemented. Transition towards a two-tier system of education in the university and the unification of specialties and areas of training with the internationally accepted educational practice dictates the need such integration (Avilova et al, 2015). Moreover, the main challenge that stands in front of the educational institutions is the task of adapting activities to the needs of market and business in order to deliver final 'products' of highest quality (Danakin et al, 2014). Currently, Russian higher education institutes are active in the field of export of educational services, both to strengthen the influence of Russia in the international community, and to increase its own international status. Since, firstly, information on the training system that makes it possible to obtain high-quality professional higher education is distributed among fellow graduates, encouraging them to make a choice in favor of the respective university. Consequently, the task of high school is that foreign students, who graduate from a Russian university, had no negative experience to pass on.

Secondly, foreign nationals who have chosen to receive professional education in the Russian higher education institutions are an important source of stable economic condition of the university, as they provide a fairly stable inflow of material resources in the form of tuition fees. The economic aspect requires special attention to the process of recruitment and subsequent training of foreign students, thus, demands the organization of activities to create a comfortable learning environment as to increase the attractiveness of the university not only in purely professional terms, but also from the socio-psychological point of view.

Thirdly, domestic students studying at the university together with the foreign citizens should be more actively involved in intercultural social processes, master the peculiarities of international communication. All of the above determines the growing interest in finding concrete ways to improve the socio-cultural adaptation of foreign citizens in the educational environment of Russian universities, as well as the study of the factors improving the management of this complex process.

\section{Literature Review}

Issues related to the peculiarities of socio-cultural adaptation of foreign students are extensively studied in modern scientific literature in different directions. For example, Shpak (1992) highlights the following components in the structure of socio-cultural adaptation that indicate its peculiarity, these are the adaptive needs, goal setting, expectations, 
aspirations, motivations, adaptive abilities, role positions and actions. Personality characteristics of students that influence the success of adaptation and mental health are tolerance, confidence, sociability and communication skills, responsibility, basic beliefs, self-organization and autonomy, self-realization, activity, values and spirituality.

For example, studies have shown that emotions play an important role in an adequate understanding of various events, and also in the adaptive response, i.e. emotions help to perceive reality and respond to it, to control the behavior and activities (Sroufe, 2005). Relevant and least explored is the issue of management of socio-cultural adaptation. The small number of scientific studies held by Londadzhim (2012), Rementsov (2011), Stefanenko (1996), Surygin (2006) are devoted to the problems of social adaptation of foreign students, most of them devoted to the study of adaptation to the pre-university stages of education (i.e. preparatory faculty). The obvious is the lack of a unified theoretical and methodological positions on the content of the main categories that describe the socio-cultural adaptation as a process and play a special role in the formulation of the principles of management of this process. Unclear is the interpretation of the object and the subject of adaptation, the concept of adaptation criteria is theoretically underdeveloped. Therefore, the aim of this paper is to address the previously mentioned issues using a case study of the Belgorod State Technological University named after V.G. Shukhov.

\section{Research Methodology}

The study is held in the Belgorod State Technological University named after V.G. Shukhov. A total number of 215 international students, 115 Russian students, 30 teachers and, 12 managers of relevant departments working with foreign students were surveyed. A questionnaire method of collecting primary sociological information was used. The empirical base of the study are the results of the survey 'Status, conditions and problems of social and cultural adaptation of foreign students', conducted by the authors in April-May 2014.

The research design was based on a system of concepts via a 'objectives tree'. The fundamental concept used was the 'adaptation readiness of foreign students'. A system of empirical indicators to be measured was developed based on the generated 'objectives tree'. Processing of the results of sociological research was carried out using the software 'DA-Systema' (version 5.0). The main mode of data processing was to obtain and visual analysis of the distribution table. When analyzing the data the methods of structural, comparative, factorial analysis, grouping and typology of social variables were used.

\section{Findings and Discussion}

The term 'rediness' is widely used in everyday conversation to indicate a condition of the subject activity. In sociology, the phenomenon of 'readiness' is not specifically studied, but rather fruitfully studied another closely related phenomenon 'social setting'. The results of these studies can be used in the conceptual and structural-functional analysis of the readiness of the social subject. They form a certain theoretical base for the expansion of research in the direction of this poorly studied phenomenon (Danakin \& Khaziev, 2013).

The social setting concept has been instrumental in the methodological reorientation of psychology. Firstly, the setting (i.e. installation) appears as a consistent state of the subject activity. Secondly, the individual is not a reagent, the behavior of which begins with the awareness of stimuli. It is always 'set' in advance, and it influences the nature of both perception and motor act. Action in psychological terms is not the reaction of the organism, but the action of the individual. Thirdly, the setting of the subject mediates its relationship with the environment, is willing to commit an act that leads to the satisfaction of needs. Fourth, notion os setting is represented with such important features of human action as the internal coherence, resistance to interference, structural stability, and organization over time.

The notion of 'readyness' (or 'willingness') is broader concept that 'setting', because it, in addition to the predisposition to certain actions, also includes preparedness for these actions (Semenova, 2003). Thanks to the concept of 'readiness', the psychologists can involve rather important phenomena of human activity and behavior into the circle of scientific analysis that have remained unaddressed previously.

Another important are of study for the sociologists, apart from social structures and processes, are the social conditions. Among them - the social status of the subject, including the state of readiness. With reference to sociology, we can formulate the following definition: willingness is a state of social subject, ensuring the successful start of operations and its subsequent execution. Readiness can be attributed to all social actors, ranging from the individual (e.g. readiness to act in an emergency situation) to the population of the country (e.g. readiness for social and economic change). Thus, readiness with regard to a particular activity serves as a stabilizing, institutional, finalizing, motivating and constructive function. 
Turning to the adaptation readiness of foreign students, we note that in the sociological literature presents some attempts to structure this type of readiness. For instance, Shapkin and Dikaya (1996) use the concept of 'resources' for the structural analysis of adapting capabilities, which characterize a set of measures, which are available or being used by the subject to ensure the efficient operation and maintenance of optimal functional state in the process of adaptation. Depending on the specific resources, it proposed to allocate four structural components of adaptation. First, the activation component associated with organic and functional costs aimed at achieving significant objectives for the subject, on the one hand, and the compensation of barrier factors that block the achievement of these objectives, on the other. Second, the cognitive component, which enables the restructuring of the cognitive system activities and is aimed at the production of the most efficient ways of processing information, without which the effective adaptation is not possible. Third, the emotional component is determined by the dynamics of emotional experiences, which are subjective indicators of effectiveness of the process of adaptation. Fourth, strong-willed (i.e. volitional) component that is represented by motivational and volitional processes, which ensure coordination of all the other components in the direction of implementation of important objectives for the subject (Shapkin and Dikaya, 1996).

Analysis of the theoretical experience in structuring adaptation readiness of a person, as well as features of the socio-cultural adaptation of foreign students defined the following categories of adaptation readiness: cognitive, informational, axiological (i.e. values), motivational, dispositional, emotional and volitional, operational, communicative, and socio-psychological.

Cognitive component reflects an understanding of foreign students of the new conditions and the arising requirements, as well as links of training with future professional activities, knowledge of the structure and content of the subject area. This element is the basis of adaptation: if a person does not understand the need to adapt and the reason of adaptation, the issue of adaptation will be removed altogether. Although it is not excluded cases where students master specific adaptation actions without a clear understanding of their meaning and purpose. Understanding is not preceded by the action, and its consequence.

Foreign students were surveyed on what better helps them to learn the language and traditions of Russia. The following answers were given (in \%): conversations in Russian language - 29.1, watching movies and TV programs 15.8 , reading -6.3 , visits to museums, festivals, excursions -5.7 . More than half of the respondents chose the answer 'all together', i.e. all of the above embodiments.

The information component of adaptation is the information availability in case of adaptation process. Foreign students can understand what to do and what to adapt to, but at the same time do not have the necessary and sufficient information for making specific decisions. The understanding here is rather a strategy of adaptation activities, while awareness is tactics. Both components are essential and interrelated. Information is useless without understanding and understanding without information is powerless.

As one of the indicators of 'informational' adaptation of foreign students had been used their 'pre-awareness' of the host country, i.e. knowledge about Russia. In this regard, the respondents were asked to answer the question about what they have heard about Russia back home. The responses were distributed as follows (see Table 1).

Table 1. Distribution of answers to the question on pre-awareness, in \%

\begin{tabular}{lccccc}
\hline \multirow{2}{*}{ Type of answer } & \multicolumn{5}{c}{ Respondent category } \\
\cline { 2 - 7 } & Africa & Asia & Europe & Latin America & Total \\
\hline Heard a lot & 32.0 & 36.1 & 22.2 & 25.0 & 31.7 \\
Heard about the harsh climate & 38.7 & 18.1 & 29.6 & 45.0 & 29.8 \\
Heard about Russian cuisine & 8.0 & 8.4 & 3.7 & - & 6.8 \\
Heard about the foreign policy of Russia & 9.3 & 18.1 & 29.6 & 20.0 & 16.6 \\
Heard about space exploration & 4.0 & 4.8 & 3.7 & - & 3.9 \\
Heard other & 5.3 & 8.4 & 3.7 & 5.0 & 6.5 \\
Heard nothing & 2.7 & 6.0 & 7.4 & - & 4.4 \\
Was interested about Russia myself & - & - & - & 5.0 & 0.5 \\
\hline
\end{tabular}

As shown in Table 1, almost all respondents (95.6\%) had heard something about the Russia back at home. Those who had no information account for just 4.4\%, and those are majority from European countries, which is rather paradoxical. Every third respondent 'heard a lot about Russia', the same number heard mostly about the harsh Russian climate. Every sixth heard of Russia's foreign policy, much less information is on 'Russian cuisine', 'space exploration' and other aspects.

Axiological component reflects the status of adaptation to the values as perceived by representations, which can 
vary between points os fanatical desire for change, the desire for this change and, no less fanatical, wishing to preserve the status quo. Some foreign citizens manifested a clear disposition to change, others were neutral - awaiting attitude ('see what happens'), the third group manifested the negative attitude (do not want to know or hear anything about change). Moreover, the negative attitudes for the upcoming organizational changes manifested in two different forms: the revaluation of the significance of change or in the form of an abstract negation.

Motivational component of adaptation expresses the desire for independence, expression of sustained interest in the subject area and the desire to fully master the language of specialty. This component means updated motives of foreign students to the change in their activities with a view to successful adaptation to the new conditions and requirements. A setting on changes related specifically to the need and defined by this need, by itself cannot determine the direction of adaptation actions. The needs derive its certainty only in the subject matter of activities: it must find itself in a person. Since the need is in the subject of their definition (objectified in it), the subject becomes the motive of activity that encourages it (Leontiev, 1971).

Moving from needs (i.e. setting) to the motive is a movement from possibility to actuality, from the abstract to the concrete, from the potential to the actual.

Foreign students have to adapt to a new language, educational, professional and cultural environment. A man can successfully go through this process, while feeling the need, being motivated. Among the motives for learning, the dominant position holds the desire to increase future social status. The second position is associated with the performance of family obligations, seeking to create the necessary starting life capital for a child. In third place is the motive of responsibility to the state, paid for training abroad. Fourth is the motive of learning desired. The first three groups of factors relate to the education as a process that can not be interrupted, leave the country, which causes psychological difficulties in development of new social and cultural environment. Successful motive that contributes to socio-cultural adaptation is the will to obtain the desired profession.

The choice of Russia as a place of education for all respondents is not associated with an interest in the culture of the country. A significant proportion of respondents (23\%) was not prepared for the trip to Russia. As many as $48 \%$ of respondents used the pre-consultation of experts, which for them were relatives and fellow compatriots already residing in Russia. Active stance on zero phase showed $22 \%$ of respondents, who, along with reccomendations, took the initiative on their own in looking at different sources of information about the country. None of the respondents had the experience of an extended stay abroad prior to the arrival to Russia.

Dispositional component. Adaptive predisposition is a concept that reflects a measure of the variability of individual behavior, which is caused by its internal readiness for self-change in response to the 'challenges' of the environment. The tendency to harmonize the requirements of the socio-cultural environment is not a constant but a variable, which can be represented as a ratio of sociocentrism (i.e. altruism) to egocentrism (i.e. selfishness). The larger the value, the more people tend to subordinate personal needs or the needs of groups of society, the sooner he accepts the norms of another culture. An extreme expression of this behavior is conformity. For conformist, it is important to establish a harmonious relationship with other people than to insist on their own principles or opinions. Ultimately, this leads to social dependence and loss of individuality. The smaller the variable of 'adaptive predisposition', the harder a person asserts his 'Myself' in a world of rapid change: most of all he values his own principles and needs rather than the interests of the group or universally valid rules of another culture. The most adequate type of person in the public interest capable of managing the changing situation and their behavior, taking into consideration not only their needs and interests, but also focusing on the changing social and spiritual foundations of life.

Emotional and volitional component characterizes the confidence of success, striving to overcome the difficulties in achieving personal goal, a high degree of self-organization, self-satisfaction from getting professionally significant information. Adaptation is a strong-willed process, its constant source is a constant inner strive to adapt to the development of the subject, being a socially determined process. The result of adaptation to change will be not only on behavioral, but also on the mental level. The adaptation of an actor can not be sufficiently judged on the basis of various behavioral characteristics, including successful solutions to problems using the existing set of decisions or actions, and the regularity of the development of stereotypes. It is necessary to take into account the internal state of the reactor, its subjective assessment of the relationship with the environment. Strictly speaking, the cultural shock is a clash of two cultures at the level of individual consciousness. This cross-cultural contact and a resulting 'cultural shock' as well as the skills produced by the person to gain control over the sotuation, are of situational nature. According to Ikonnikova (1995), this means that by adapting to work in one culture, a person will have difficulty falling in an informal setting, moving to another country, or even back home, since the experience gained will change its orientation, and a forced gap of public relations at home will require re-approval of their social status (Ikonnikova, 1995).

The operational component expresses the possession of a linguistic apparatus for professional mastering of 
important information, possession of skills of independent learning activities.

Obviously, staying in another country, especially the first time relates to the 'language' issues. Answering the question 'In what language did you speak the first time in Russia?', more than half of respondents (56.3\%) answered 'in English', 36.1\% - 'the mother tongue' and only 1.9\% - 'in Russian'. Part of foreing students (5.7\%) states that 'it was difficult because I did not know English'. Foreign students are also interviewed on the subject of the difficulties caused by their lack of knowledge of the Russian language. These difficulties are as follows (percentage of respondents): difficulty in communicating with people - 61.4; difficulties in the bank - 26.6; difficulties in the city - 17.1; difficulties in public transport - 15.2; difficulties in the store - 12.7. Only $3.8 \%$ of respondents said that they had 'no difficulty'. As it turned out, only $12.7 \%$ of the respondents studied the Russian language at home, 17.7\% - 'a little studied', $69.6 \%$ - 'not studied'.

The communicative component of socio-cultural adaptation is the establishment and strengthening of mutual understanding of foreigners in terms of revaluation and the logic of the usual (traditional) performances and installations. Foreign students are not a set of isolated people, but a group of students, combined by joint actions, common, or at least mutually agreed goals and interests, a similar understanding of these things and mutual understanding. Therefore, the formation and development of their communicative community is critical for adaptation.

Socio-psychological adaptation component expresses the unity and consolidation of the students, both with faculty and fellow students for a successful solution of adaptation problems, therefore, a positive change in their internal environment. Without this element, the process of socio-cultural adaptation is not only incomplete but also impracticable.

By concluding the discussion on the structure of adaptation readiness of foreign students, one shall pay attention to the importance of adhering to the principles of the system and moderately.

Compliance with the systemic principle relates to the fact that the process of adaptation of foreign students to the new socio-cultural environment is structurally complex and differentiated. It is characterized by the fact that the implementation and the development of personal potential, relative adaptation to the new socio-cultural environment of foreigners is achieved solely by changes in stereotypes and ways of behavior. Yet, this is an internal alienation from the social environment in which they are forced to live and study for five or seven years. The systematic study of the particularities in implementation and the development of personal potential, as well as socio-cultural adaptation of students from foreign countries living and studying in another country could help to optimize the system of higher education that, in the end, is designed to support the international prestige of the country, confirming the high level of science, culture and education. In addition, analysis of the social adaptation of foreign students and the subsequent practical advice developed on its basis on the preparation and introduction of adaptation measures will improve the competitiveness of Russian universities in the field of international educational services, which will, in turn, contribute to enhancing the professional employment of highly qualified faculty members in Russian universities and the development of infrastructure of higher education in Russia.

The principle of moderately involves the allocation of the limit beyond the boundaries of which a quantify measure of reaction, a change in the strategy of behavior, plasticity of living and thinking lead to a qualitative transformation of the valuable core of the culture. The moderately of socio-cultural adaptation is set by qualitative limits of existence of this culture. Within the limits of moderately, the improvement of the old and the emergence of new activities, new forms of response to changes in the environment contribute to the progressive development of society. The moderately of sociocultural adaptation is the most important regulative of harmonization, of changes and stabilization of the society. It optimizes the social behavior of individuals, legitimizes the direction and nature of innovative changes in the public consciousness through the adoption of dynamic integrity of the norms and value structure. The latter includes the lower limit (i.e. the norms of everyday utilitarian life) and the top (i.e. absolute values, ideals). The intensity, nature and depth of the adaptive process can be locked in this 'scale' according to the approximation to the lower norm or upper ideal state. Violation moderately state leads to negative, destructive consequences for society and for the individual himself, up to the emergence of the phenomenon of 'destructive adaptation' (Makhanko, 2001).

\section{Concluding Remarks}

Readiness is a state of social subject that ensures the successful start of operations and its subsequent execution. Readiness in relation to an activity executes stabilizing, institutional, finalizing, motivating and constructive functions. Analysis on structuring of adaptation readiness of a person, as well as features of socio-cultural adaptation of foreign students enabled to identify the following components of adaptation readiness of foreign students: cognitive, informational, evaluative, motivational, dispositional, emotional, volitional, operational, communicative, and sociopsychological. Results of the study enabled the authors to discover the correlation of these components of adaptation readiness of foreign students. Meanwhile, the authors come to a conclusion about the importance of compliance with the 
systemic and moderately principles in managing the socio-cultural adaptation of foreign students.

\section{References}

Avilova, Z.N., Gulei, I.A., \& Shavyrina, I.V. (2015). Formation of the customer-centric organizational culture of the university as a factor of effective social and economic development of the region. Mediterranean Journal of Social Sciences, 6 (3), 207-216.

Danakin, N., Shutenko, A., \& Ospishev, P. (2014). Humanitarian measurements of innovative development of the higher school. International Journal of Applied Engineering Research, 9(22), 16937-16944.

Danakin, N.S., \& Khaziev, I.H. (2013). Behavioral trajectory in a situation of social threat. Proceedings of the higher educational institutions. Volga region. Social Sciences, 3 (27), 92-99.

Ikonnikova, N.K. (1995). Mechanisms for inter-cultural perceptions. Sociological Research, 11, 26-34.

Leontiev, A.N. (1971). Problems of development of the psyche. Moscow: MGU.

Londadzhim, T. (2012). The socio-cultural adaptation of foreign students studying in Russian universities. PhD thesis. Nizhny Novgorod State University. NI Lobachevsky. Nizhny Novgorod.

Makhanko, N.V. (2001). The socio-cultural adaptation of the transformation processes in culture. PhD thesis. Rostov State University. Krasnodar.

Rementsov, A.N. (2011). Sociocultural aspects of the adaptation of foreign students in the Russian higher technical educational institutions. Bulletin of high school, 7, 10-14.

Semenova, TY (2003). Dynamics of formation of readiness of graduates for professional careers in the modern liberal arts college: sociological aspect. Law and Education, 2.

Shapkin, S.A., \& Dikaya, L.G. (1996). Activities in special cases: component analysis of the structure and adaptation strategies. Psychological Journal, 1, 19-34.

Shpak, L.L. (1992). The socio-cultural adaptation: the nature, direction, implementation mechanism. Doctoral thesis of Social Sciences. Moscow.

Sroufe, LA. (2005). Attachment and development: a prospective, longitudinal study from birth to adulthood. Attachment \& Human Development, 7(4), 349-67.

Stefanenko, T.G. (1996). Adapting to a new culture and ways of its optimization. Introduction to practical social psychology. Moscow: Smisl.

Surygin, A.I. (2006). Pedagogical designing of pre-university training of foreign students. SPb: Zlatoust. 